\title{
Achieving Scalability and Expressiveness in an Internet-Scale Event Notification Service
}

\author{
Antonio Carzaniga \\ Dept. of Computer Science \\ University of Colorado \\ Boulder, CO 80309-0430 \\ USA \\ carzanig@cs.colorado.edu
}

\author{
David S. Rosenblum \\ Dept. of Information \& \\ Computer Science \\ University of California, Irvine \\ Irvine, CA 92697-3425 \\ USA \\ dsr@ics.uci.edu
}

\author{
Alexander L. Wolf \\ Dept. of Computer Science \\ University of Colorado \\ Boulder, CO 80309-0430 \\ USA \\ alw@cs.colorado.edu
}

\begin{abstract}
This paper describes the design of SIENA, an Internet-scale event notification middleware service for distributed eventbased applications deployed over wide-area networks. SIENA is responsible for selecting the notifications that are of interest to clients (as expressed in client subscriptions) and then delivering those notifications to the clients via access points. The key design challenge for SIENA is maximizing expressiveness in the selection mechanism without sacrificing scalability of the delivery mechanism. This paper focuses on those aspects of the design of SIENA that fundamentally impact scalability and expressiveness. In particular, we describe SIENA's data model for notifications, the covering relations that formally define the semantics of the data model, the distributed architectures we have studied for $S$ IENA's implementation, and the processing strategies we developed to exploit the covering relations for optimizing the routing of notifications.
\end{abstract}

\section{INTRODUCTION}

There is a clear trend among experienced software developers toward designing large-scale distributed systems as assemblies of loosely-coupled autonomous components. A common approach to achieving loose coupling is an eventbased or implicit invocation design style [7]. In an eventbased system, component interactions are modeled as asynchronous occurrences of, and responses to, events. To inform other components about the occurrences of internal events (such as state changes), components emit notifications containing information about the events. Upon receiving notifications, other components can react by performing actions that, in turn, may result in the occurrence of other events and the generation of additional notifications.

Wide-area networks such as the Internet, with their vast

Permission to make digital or hard copies of all or part of this work for personal or classroom use is granted without fee provided that copies are not made or distributed for profit or commercial advantage and that copies bear this notice and the full citation on the first page. To copy otherwise, to republish, to post on servers or to redistribute to lists, requires prior specific permission and/or a fee.

PODC 2000 Portland Oregon

Copyright ACM 2000 1-58113-183-6/00/07...\$5.00 number of potential producers and consumers of notifications, create an opportunity for developing novel distributed event-based applications in such fields as market analysis, data mining, indexing, and security. In general, the asynchrony, heterogeneity, and inherent high degree of loose coupling that characterize applications for wide-area networks suggest event interaction as a natural design abstraction for a growing class of distributed systems. Yet to date there has been a lack of sufficiently powerful and scalable middleware infrastructures to support event-based interaction in a widearea network. We refer to such a middleware infrastructure as an event notification service [16].

This paper describes the design of $S_{\text {IENA }}{ }^{1}$ an Internet-scale event notification service that is representative of the capabilities we envision for scalable event notification middleware. SIENA is designed to be a ubiquitous service accessible from every site on a wide-area network. As shown in Figure 1, SIENA is implemented as a distributed network of

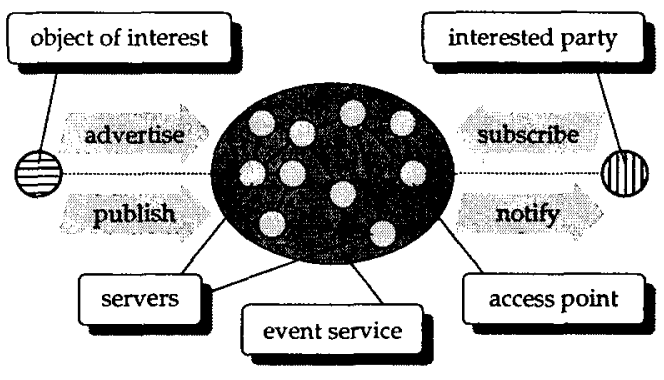

Figure 1: Distributed Event Notification Service.

servers that provide clients with access points offering an extended publish/subscribe interface. The clients are of two kinds: objects of interest, which are the generators of notifications, and interested parties, which are the consumers of notifications; of course, a client can act as both an object of interest and an interested party. Clients use the access points of their local servers to advertise the information about notifications that they generate and to publish the advertised notifications. Clients also use the access points to subscribe for individual notifications or compound patterns

\footnotetext{
${ }^{1}$ Scalable Internet Event Notification Architectures.
} 
of notifications of interest. SIENA is responsible for selecting the notifications that are of interest to clients and then delivering those notifications to the clients via the access points.

SIENA is a best-effort service in that it does not attempt to prevent race conditions induced by network latency. This is a pragmatic concession to the realities of Internet-scale services, but it means that clients of $S$ IENA must be resilient to such race conditions. For instance, clients must allow for the possibility of receiving a notification for a cancelled subscription. Of course, an implementation would likely adopt techniques such as persistent data structures, transactional updates to the data structures, and reliable communication protocols to enhance the robustness of this best-effort service.

The key design challenge we face in supporting event notification of this kind in a wide-area network is maximizing expressiveness in the selection mechanism without sacrificing scalability of the delivery mechanism. Scalability refers not only to the numbers of publishers and subscribers, and the numbers of notifications and subscriptions, but also to the need to discard many of the assumptions made for local-area networks, such as low latency, abundant bandwidth, homogeneous platforms, continuous and reliable connectivity, and centralized control. Expressiveness refers to the power of the data model that is offered to publishers and subscribers of notifications. Clearly the level of expressiveness influences the algorithms used to route and deliver notifications, and the extent to which those algorithms can be optimized. As the power of the data model increases, so does the complexity of the algorithms. Therefore, the expressiveness of the data model ultimately influences the scalability of the implementation, and hence scalability and expressiveness are two conflicting goals that must be traded off.

While we have not fully explored the nature of this tradeoff, we have investigated a number of carefully chosen points in the tradeoff space. In particular, we designed a data model for $S$ IENA that we believe is sufficiently expressive for a wide range of applications while still allowing sufficient scalability of the delivery mechanism. Based on this data model, we designed distributed server architectures and associated delivery algorithms and processing strategies, and we evaluated and confirmed their scalability. Our description of $S$ IENA in this paper focuses on those aspects of the design that fundamentally impact expressiveness and scalability.

Section 2 presents the data model of notifications in SIENA. Section 3 presents the semantics of $S$ IENA, which is described formally in terms of covering relations over advertisements, subscriptions and notifications. Section 4 describes the alternative architectures for SIENA that we have studied and their associated processing strategies; the processing strategies exploit the covering relations for purposes of optimizing the routing of notifications. Section 5 presents an analysis of the complexity of the algorithms, demonstrating the scalability of $S$ IENA with respect to the level of expressiveness we chose for its data model. Section 6 concludes with a discussion of related work, a brief description of a prototype implementation of $S$ IENA, and a discussion of some of our plans for future work.

\section{DATA MODEL}

As mentioned above, $S$ IENA extends the traditional publish/subscribe protocol with an additional interface function called advertise, which is used by an object of interest to inform the event service of the nature of the notifications that it might publish. SIENA also adds the functions unsubscribe and unadvertise to further inform the event service about the future behavior of interested parties and objects of interest, respectively. Subscriptions can be matched repeatedly until they are cancelled by a call to unsubscribe. Advertisements remain in effect until they are cancelled by a call to unadvertise.

Underlying $S$ IENA's interface is a notification data model (or simply data model) that drives the semantics of the service. A notification in the model is an untyped set of typed attributes. For example, the notification shown in Figure 2 represents a stock price change event. Each individual at-

\begin{tabular}{|lrl|}
\hline string & class & $=$ finance/exchanges/stock \\
time & date & $=$ Mar 4 11:43:37 MST 1998 \\
string & exchange & $=$ NYSE \\
string & symbol & $=$ DIS \\
float & prior & $=105.25$ \\
float & change & $=-4$ \\
float & earn & $=2.04$ \\
\hline
\end{tabular}

Figure 2: Example of a Notification.

tribute has a type, a name, and a value, but the notification as a whole is purely a structural value derived from its attributes. Attribute names are simply character strings. The attribute types belong to a predefined set of primitive types commonly found in programming languages and database query languages, and for which a fixed set of operators is defined.

The justification for choosing this typing scheme is scalability: Typed notifications, such as one finds for example in the Java Distributed Event Specification [19] and CORBA Notification Service [14], imply a global authority for managing and verifying the type space, something which is clearly not feasible at an Internet scale. On the other hand, we define a restricted set of attribute types from which to construct (arbitrary) notifications. By having this well-defined set, we can perform efficient routing based on the content of notifications. Content-based routing is a powerful technique for selecting and delivering notifications that gives clients the ability to control the precision with which notifications are selected and gives the event service the ability to optimize the processing tasks required for notification delivery. As we discuss in Section 5 and Section 6, content-based routing has distinct advantages over the alternative schemes of channel- and subject-based routing.

In the remainder of this section we discuss two mechanisms for notification selection, namely filters and patterns, that form the essence of $S$ IENA's extended publish/subscribe protocol. This allows us to fully define the semantics of the interface functions, which we do in Section 3 in terms of what we call covering relations. In Section 4 we discuss the use of the covering relations to define the processing strategies 
that lead to optimized notification delivery.

An event filter, or simply a filter, selects event notifications by specifying a set of attributes and constraints on the values of those attributes. Each attribute constraint is a tuple specifying a type, a name, a binary predicate operator, and a value for an attribute. The operators provided by SIENA include all the common equality and ordering relations $(=$ $\neq,<$,$\rangle , etc.) for each of its types, substring (*), prefix$ $(>*)$, and suffix $(*<)$ operators for strings, and an operator any that matches any value.

An attribute $\alpha=\left(\right.$ type $_{\alpha}$, name $_{\alpha}$, value $\left._{\alpha}\right)$ matches an attribute constraint $\phi=\left(\right.$ type $_{\phi}$, name $_{\phi}$, operator $_{\phi}$, value $\left._{\phi}\right)$ iff type $_{\alpha}=$ type $_{\phi} \wedge$ name $_{\alpha}=$ name $_{\phi} \wedge$ operator $_{\phi}\left(\right.$ value $_{\alpha}$, value $\left._{\phi}\right)$. We say an attribute $\alpha$ satisfies or matches an attribute constraint $\phi$ with the notation $\phi \sqsubset \alpha$. When $\alpha$ matches $\phi$, we also say that $\phi$ covers $\alpha$. Figure 3 shows a filter that matches price decreases for stock DIS on stock exchange NYSE.

\begin{tabular}{||l} 
string class $>*$ finance/exchanges/| \\
string exchange $=$ NYSE \\
string symbol $=$ DIS \\
float change $<0$
\end{tabular}

Figure 3: Example of an Event Filter.

While a filter is matched against a single notification based on the notification's attribute data, a pattern is matched against one or more notifications based on both their attribute data and on the combination they form. At its most generic, a pattern might correlate events according to any compound relation. For example, the programmer of a stock market analysis tool might be interested in receiving price change notifications for the stock of one company only if the price of a related stock has changed by a certain amount. Rich languages and logics exist that allow one to express event patterns [12].

We do not attempt to provide a complete pattern language. Our goal is rather to study pattern operators that can be exploited to optimize the selection of notifications within the event service. Here, we restrict a pattern to be syntactically a sequence of filters, $f_{1} \cdot f_{2} \cdots f_{n}$, that is matched by a temporally ordered sequence of notifications, each one matching the corresponding filter. An example of a pattern is shown in Figure 4, which matches an increase in the price of stock MSFT followed by a subsequent increase in the price of stock NSCP. In general, we observe that more

\begin{tabular}{l}
$\mid \begin{array}{l}\text { string what }>* \text { finance/exchanges/| } \\
\text { string symbol }=M S F T \\
\text { float change }>0\end{array}$ \\
$\bullet$ \\
$\begin{array}{l}\text { string what }>* \text { finance/exchanges/ } \\
\text { string symbol }=N S C P \\
\text { float change }>0\end{array}$ \\
\hline
\end{tabular}

Figure 4: Example of an Event Pattern. sophisticated forms of patterns can always be split into a set of simple subscriptions and then matched externally to SIENA (i.e., at the access point of the subscriber), although this is likely to induce extra network traffic.

Since patterns involve matching of multiple events occurring potentially in different parts of a network, latency effects influence the semantics of the pattern operators. In accordance with SIENA's best-effort semantics, notifications are given timestamps indicating when they were published. ${ }^{2}$ This allows the service to detect and account for the effects of latency on the matching of patterns, which means that within certain limits the actual order of notifications can be recognized.

\section{COVERING RELATIONS}

When a filter is used in a subscription, multiple constraints for the same attribute are interpreted as a conjunction; all such constraints must be matched. Thus, we say that a notification $n$ matches a filter $f$, or equivalently that $f$ covers $n\left(f \sqsubset_{S}^{N} n\right.$ for short):

$$
f \sqsubset_{S}^{N} n \Leftrightarrow \forall \phi \in f: \exists \alpha \in n: \phi \sqsubset \alpha
$$

Notice that the notification may contain other attributes that have no correspondents in the filter. Table 1 gives some examples that illustrate the semantics of $\sqsubset_{S}^{N}$. The second

\begin{tabular}{|c|c|c|}
\hline \multicolumn{2}{|l|}{ subscription } & notification \\
\hline string what = alarm & $\sqsubset \sqsubset_{S}^{N}$ & 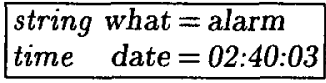 \\
\hline 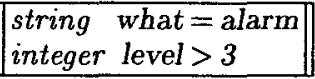 & $\not \subset S$ & $\begin{array}{l}\text { string } \text { what }=\text { alarm } \\
\text { time date }=02: 40: 03\end{array}$ \\
\hline $\begin{array}{|ll|}\text { string } & \text { what }=\text { alarm } \\
\text { integer } & \text { level }>3 \\
\text { integer } & \text { level }<7\end{array}$ & $\not_{L}^{N}$ & $\begin{array}{l}\text { string what }=\text { alarm } \\
\text { integer level }=10\end{array}$ \\
\hline $\begin{array}{|ll|}\text { string } & \text { what }=\text { alarm } \\
\text { integer } & \text { level }>3 \\
\text { integer } & \text { level }<7\end{array}$ & $\sqsubset_{S}^{N}$ & \begin{tabular}{|l} 
String what $=$ alarm \\
integer level $=5$
\end{tabular} \\
\hline
\end{tabular}

Table 1: Examples of $\sqsubset_{S}^{N}$.

example is not a match because the notification is missing a value for attribute level. The third example is not a match because the constraints specified for attribute level in the subscription are not matched by the value for level in the notification.

We define the semantics of advertisements with a similar relation $\subset_{A}^{N}$. The motivation for advertisements is to inform the event service about which kind of notifications will be generated by which objects of interest, so that it can

${ }^{2}$ With the advent of accurate network time protocols and the existence of the satellite-based Global Positioning System (GPS), it is reasonable to assume the existence of a global clock for creation of these timestamps, and it is hence reasonable for all but the most time-sensitive applications to rely on these timestamps. 
best direct the propagation of subscriptions. The idea is that, while a subscription defines the set of interesting notifications for an interested party, an advertisement defines the set of notifications potentially generated by an object of interest. Therefore, the advertisement is relevant to the subscription only if these two sets of notifications have a non-empty intersection.

The relation $\complement_{A}^{N}$ defines the set of notifications covered by an advertisement:

$$
a \sqsubset_{A}^{N} n \Leftrightarrow \forall \alpha_{n} \in n: \exists \phi_{a} \in a: \phi_{a} \sqsubset \alpha_{n}
$$

This expression says that an advertisement covers a notification if and only if it covers each individual attribute in the notification. Note that this is the dual of subscriptions, which define the minimal set of attributes that a notification must contain. In contrast to subscriptions, when a filter is used as an advertisement, then multiple constraints for the same attribute are interpreted as a disjunction rather than as a conjunction; only one of the constraints need be satisfied. Table 2 shows some examples of the relation $\sqsubset_{A}^{N}$. The second example is not a match because the attribute

\begin{tabular}{|c|c|c|}
\hline \begin{tabular}{|l|} 
advertisement \\
string what $=$ alarm \\
string what $=$ login \\
string user any
\end{tabular} & $ᄃ_{A}^{N}$ & $\begin{array}{l}\text { notification } \\
\text { string what = alarm }\end{array}$ \\
\hline $\begin{array}{l}\text { string what = alarm } \\
\text { string what = login } \\
\text { string user any }\end{array}$ & $\not \not_{A}^{N}$ & $\begin{array}{l}\text { string what }=\text { alarm } \\
\text { time date }=02: 40: 03\end{array}$ \\
\hline $\begin{array}{l}\text { string what }=\text { alarm } \\
\text { string what }=\text { login } \\
\text { string user any }\end{array}$ & $\sqsubset_{A}^{N}$ & $\begin{array}{l}\text { string what = login } \\
\text { string user = carzanig }\end{array}$ \\
\hline $\begin{array}{l}\text { string what }=\text { alarm } \\
\text { string what }=\text { login } \\
\text { string user any }\end{array}$ & $\not Z_{A}^{N}$ & $\begin{array}{l}\text { string what = logout } \\
\text { string user = carzanig }\end{array}$ \\
\hline
\end{tabular}

Table 2: Examples of $\leftarrow_{A}^{N}$.

date of the notification is not defined in the advertisement. The fourth example is not a match because the value of attribute what in the notification does not match any of the constraints defined for what in the advertisement.

So far we have defined a number of relations that express the semantics of subscriptions and advertisements:

- $\phi \sqsubset \alpha$ : attribute $\alpha$ matches attribute constraint $\phi$;

- $f \sqsubset_{S}^{N} n$ : notification $n$ matches filter $f$, where $f$ is interpreted as a subscription filter;

- $a \sqsubset_{A}^{N} n$ : notification $n$ matches filter $a$, where $a$ is interpreted as an advertisement filter;

From these, other relations can be derived:
- $f_{1} \sqsubset_{S}^{S} f_{2}$ : filter $f_{1}$ covers filter $f_{2}$, where $f_{1}$ and $f_{2}$ are interpreted as subscriptions. Formally,

$$
f_{1} \sqsubset_{S}^{S} f_{2} \Leftrightarrow \forall n: f_{2} \sqsubset_{S}^{N} n \Rightarrow f_{1} \sqsubset_{S}^{N} n
$$

which means that $f_{1}$ covers a superset of the notifications covered by $f_{2}$.

- $a_{1} \sqsubset_{A}^{A} a_{2}$ : filter $a_{1}$ covers filter $a_{2}$, where $a_{1}$ and $a_{2}$ are interpreted as advertisements. Formally:

$$
a_{1} \sqsubset_{A}^{A} a_{2} \Leftrightarrow \forall n: a_{2} \sqsubset_{A}^{N} n \Rightarrow a_{1} \sqsubset_{A}^{N} n
$$

which means that $a_{1}$ covers a superset of the notifications covered by $a_{2}$.

The relations $\complement_{S}^{S}$ and $\sqsubset_{A}^{A}$ can also define the equality relation between filters with its intuitive meaning:

$$
f=g \Leftrightarrow g \sqsubset f \wedge f \sqsubset g
$$

In the next section we describe how we exploit these derived relations in $S$ IENA's processing strategies.

Unsubscriptions and unadvertisements cancel previous subscriptions and advertisements, respectively. These operations must be performed in the context of the covering relations so that the proper subscriptions and advertisements remain in place. The details of this are complex and are described elsewhere $[2,3]$.

\section{ARCHITECTURES AND PROCESSING STRATEGIES}

As shown in Figure 1, the implementation of $S$ IENA comprises a number of interconnected servers, ${ }^{3}$ each serving some subset of the clients of the service. In effect, $S_{\text {IENA }}$ is a wide-area network of pattern matchers and routers overlaid atop some other wide-area communication facility, such as the Internet. One reasonable allocation of such servers might be to place a server at each administrative domain within the low-level, wide-area communication network.

Creating a network of servers to provide a distributed service of any sort gives rise to three critical design issues:

- Interconnection topology. In what configuration should the servers be connected?

- Routing algorithm. What information should be communicated between the servers to allow the correct and efficient delivery of messages?

- Processing strategy. Where in the network, and according to what heuristics, should message data be processed in order to optimize message traffic?

These three design issues have been studied extensively for many years and in many contexts. Our challenge is to find a solution in the particular domain of Internet-scale event notification, leveraging previous results (both positive and negative) wherever possible.

${ }^{3}$ Note that some authors use the term proxy or broker instead of server for this concept. 
A pair of interconnected servers use a server/server communication protocol that determines what kinds of information they can exchange, and in which direction. This protocol might make use of any one of a number of lower-level network protocols, such as SMTP or HTTP, through standard encoding and/or tunneling techniques. An interconnection topology and a protocol together define what we refer to as an architecture for SIENA. We have studied three basic architectures for SIENA: hierarchical client/server, acyclic peer-to-peer, and general peer-to-peer. We also have studied some hybrids of these three architectures. Because it is not scalable, we ignore the degenerate case of a centralized architecture having a single server.

In the hierarchical client/server architecture, the servers form a hierarchical topology, with each server (except the root server) behaving like a $S$ IENA client of the server one level up the hierarchy. As we have demonstrated elsewhere [3], the main problems exhibited by the hierarchical architecture are the potential overloading of servers high in the hierarchy and the fact that each server is a single point of failure. In the acyclic peer-to-peer architecture, servers communicate with each other symmetrically as peers in an acyclic undirected graph, adopting a protocol that allows a bi-directional flow of subscriptions, advertisements, and notifications. Removing the constraint of acyclicity from the acyclic peer-to-peer architecture, we obtain the general peer-to-peer architecture, which can have multiple paths of bi-directional communication between servers. Allowing redundant connections makes it more robust with respect to failures of single servers. The drawback of having redundant connections is that special algorithms must be implemented to avoid cycles and to choose the best paths. These three basic architectures can be combined to form hybrid architectures, such as an acyclic peer-to-peer topology of subnets, each subnet being a hierarchy.

Once a topology of servers is defined, they must establish appropriate routing paths to ensure that notifications published by an object of interest are correctly delivered to all the interested parties that subscribed for them. In general, we observe that notifications must "meet" subscriptions somewhere in the network so that the notifications can be selected according to the subscriptions and then dispatched to the subscribers. This common principle can be realized according to a spectrum of possible routing algorithms. One possibility is to maintain subscriptions at their access point and to broadcast notifications throughout the whole network; when a notification meets and matches a subscription, the subscriber is immediately notified locally. However, since we expect the number of notifications to far exceed the number of subscriptions or advertisements, this strategy appears to offer the least possible efficiency, and so we consider it no further for SIENA.

To devise more efficient routing algorithms, we employ principles found in IP multicast routing protocols [6]. Similar to these protocols, the main idea behind the routing algorithms of $S$ IENA is to send a notification only towards event servers that have clients that are interested in that notification, possibly using the shortest path. The same principle applies to patterns of notifications as well. More specifically, we formulate two generic principles that become requirements for our routing algorithms:

downstream replication: A notification should be routed in one copy as far as possible and should be replicated only downstream, that is, as close as possible to the parties that are interested in it.

upstream evaluation: Filters are applied and patterns are assembled upstream, that is, as close as possible to the sources of (patterns of) notifications.

These principles are implemented by two classes of routing algorithms, the first of which involves broadcasting subscriptions and the second of which involves broadcasting advertisements:

subscription forwarding: In an implementation that does not use advertisements, the routing paths for notifications are set by subscriptions, which are propagated throughout the network so as to form a tree that connects the subscribers to all the servers in the network. When an object publishes a notification that matches that subscription, the notification is routed towards the subscriber, following the reverse path put in place by the subscription.

advertisement forwarding: In an implementation that uses advertisements, it is safe to send a subscription only towards those objects of interest that intend to generate notifications relevant to that subscription. Thus, advertisements set the paths for subscriptions, which in turn set the paths for notifications. Every advertisement is propagated through the network, thereby forming a tree that reaches every server. When a server receives a subscription, it propagates the subscription in reverse, along the path to the advertiser, thereby activating that path. Notifications are then forwarded only through the activated paths.

In the simplest implementation of the subscription forwarding (advertisement forwarding) algorithms, all subscriptions (advertisements) would be stored at all servers. However, we can optimize their implementation by exploiting the covering relations defined in Section 3. When a subscription reaches a server (either from a client or from another server), the server propagates that subscription only if it defines new selectable notifications that are not in the set of selectable notifications defined by any previously propagated subscription. Three benefits accrue from this approach: First, we reduce network costs by pruning the propagation of new subscriptions. Second, we reduce the storage requirements for servers. Third, by reducing the number of subscriptions held at each server, we reduce the computational resources needed to match notifications at that server. We use a similar strategy for propagation of advertisements.

In order to keep track of the propagation of subscriptions (and similarly advertisements), every server maintains a partially ordered set (poset) of subscriptions, where the partial order relation is defined by the covering relations. Each server associates with each subscription $s$ a set of subscribers 
subscribers $(s)$ and a set of neighbor servers $f w d s(s)$ to which $s$ has been forwarded. $f w d s(s)$ is a subset of neighbors, the set of all neighbors of the server in the network.

When a server receives a subscription $f$ from a client or a neighbor server $U$, it looks in its subscriptions poset $P_{S}$ for either

1. a subscription $f^{\prime}$ that covers $f$ and has $U$ among its subscribers: $f^{\prime} \complement_{S}^{S} f \wedge U \in$ subscribers $\left(f^{\prime}\right)$. In this case, the procedure that handles the subscription returns with no effect; or

2. a subscription $f^{\prime}$ that is equal to $f$ and does not contain $U$ in its subscribers: $f^{\prime} \sqsubset_{S}^{S} f \wedge f \sqsubset_{S}^{S} f^{\prime}$. Here the server adds $U$ to subscribers $\left(f^{\prime}\right)$; or

3 . two possibly empty sets $\bar{f}$ and $f$, representing the immediate predecessors and the immediate successors of $f$ respectively. Here the server inserts $f$ as a new subscription between $\bar{f}$ and $f$, and adds $U$ to subscribers $(f)$.

In cases 2 and 3 , the server also removes $U$ from all the subscriptions in $P_{S}$ that are covered by $f$, and then removes from $P_{S}$ those subscriptions that have no other subscribers.

Next, the server forwards the subscription to some of its neighbors. Formally, given a subscription $f$ in $P_{S}$, let $f w d s(f)$ be defined as follows:

$$
f w d s(f)=\text { neighbors }-N S T(f)-\bigcup_{f^{\prime} \in P_{S} \wedge f^{\prime} \complement_{S}^{S} f} f w d s\left(f^{\prime}\right)
$$

In other words, $f$ is forwarded to all neighbors except those not on any spanning tree rooted at an original subscriber of $f$ (the second term in the formula), and those to which subscriptions $f^{\prime}$ covering $f$ have been forwarded already by this server (the last term in the formula).

The last term in the formula represents the optimization that the server can make in the situation where more generic subscriptions have been propagated already to some neighbors.

Hence, in the process of forwarding subscriptions or advertisements, $S$ IENA exploits commonalities among subscriptions or advertisements. In practice, SIENA prunes the propagation trees by following only those paths that have not been covered by previous requests. The derived covering relations $\sqsubset_{S}^{S}$ and $\sqsubset_{A}^{A}$ defined in Section 3 are used to determine whether a new subscription or a new advertisement is covered by a previous one that has already been forwarded.

Unsubscriptions and unadvertisements are handled in a similar way to undo the effect of the affected subscriptions or advertisements.

To match patterns, servers assemble sequences of notifications from small subsequences or from single notifications according to the advertised paths along which notifications will be propagated. For this reason, advertisement forwarding algorithms are necessary to implement the upstream evaluation principle for event patterns.
The interested reader is referred to our other publications [2, 3 ] for details on how we apply these processing strategies to the different architectures.

\section{ANALYSIS}

Consider two extremes of expressiveness. In a channel-based model of event notification, notifications are fed into what amounts to a discrete communications pipe. Subscriptions are made by simply identifying the pipe (i.e., channel) from which notifications are expected to flow; the notion of "filtering" then reduces to channel selection. Since the contents of notifications are not used in routing, it is not necessary to define any service-visible structure within notifications. The covering relations become an equality check on the identifier of the channel, thus making the routing of notifications very efficient. However, the resulting notification selection mechanism is simplistic, and too weak for some applications.

At the opposite extreme, the structure of notifications, the types of attributes within notifications, and the operators that can be applied to those attributes are all application defined, perhaps employing the full expressive power of a Turing-complete language. However, the operators, which are used by the service to perform notification selection, would then be of an arbitrary, unknown, and potentially unbounded complexity. Moreover, the computation of the covering relations that allow the pruning of propagation trees, such as $\complement_{S}^{S}$, might be undecidable.

These considerations led us to a level of expressiveness in SIENA at which notification structure, attribute types, and attribute operators approximate those of the wellunderstood and widely-used database query language SQL In particular, $S$ IENA supports the definition of filters that essentially implement a significant subset of the SQL select query.

The covering relations are well behaved and predictable in the sense that they exhibit an arguably reasonable computational complexity deriving from the expressiveness of filters: Assuming a brute-force and unoptimized algorithm, the complexity of computing $\left\ulcorner_{S}^{N}\right.$ on a given subscription and a given notification is $O(n+m)$, where $n$ is the number of attribute constraints in the subscription filter and $m$ is the number of attributes in the notification. The complexity of each individual comparison is $O(1)$ for all the predefined types we have included in SIENA. The only exception is for the string type, but efficient comparison algorithms are well known. The complexity of computing $\complement_{S}^{N}$ reflects the computation of an intersection between the attribute values in a notification and constraints on those values appearing in a subscription.

The complexities of computing $\complement_{S}^{S}$ and $\sqsubset_{A}^{A}$ are all $O(n m)$, where $n$ and $m$ represent the number of attribute constraints appearing in the respective subscription and/or advertisement filters. This complexity represents a comparison between each attribute constraint in one filter and any corresponding attribute constraints in the other filter. Checking a covering relation between filters amounts to a universal quantification. But given our choice of types and operators, comparing a pair of attribute constraints can be reduced to evaluating an appropriate predicate on the two constant val- 
ues of the constraints, with a complexity $O(1)$. For example, to see if $\left[\mathrm{x}>k_{1}\right]$ covers $\left[\mathrm{x}>k_{2}\right]$ we can simply verify that $k_{2} \geq k_{1}$.

We also restricted the expressiveness of patterns in SIENA in the interests of efficiency. As we discuss in Section 2 , patterns are simple sequences of filters. The computational complexity of recognizing a pattern is $O(l(n+m))$, where $l$ is the length of the pattern. This means that it is linear in the number of filters, whose covering relation $\complement_{S}^{N}$ has complexity $O(n+m)$.

Our conclusion from this analysis is that the optimizations presented in Section 4 are effective, since they derive from the reasonable complexity of the covering relation computations. In fact, the factors $n$ and $m$ are, in practice, likely to be relatively small (typically less than 10), making the computations negligible compared to the network costs they are attempting to reduce. This is all achieved with an expressiveness that approximates SQL.

With respect to the scalability of the service across a distributed network, we have carried out simulation studies to determine how the architectures and processing strategies perform over an extensive range of scenarios having different network configurations and application behaviors. Details of the simulation framework and specific results are available elsewhere $[2,3]$.

\section{CONCLUSION}

In this section we briefly review related work in event notification services and discuss our prototype implementation of $S$ IENA. A more complete discussion of these topics is presented elsewhere $[2,3]$.

We can compare related technologies from the perspective of their server architecture, which affects scalability, and from the perspective of their subscription language, which determines expressiveness. Table 3 presents such a comparison in terms of the architectures described in Section 4 and in terms of a classification of subscription languages shown in Table 4

We classify subscription languages based on their scope and expressive power. Scope has two aspects: (1) whether a subscription is limited to considering a single notification (thus reducing the language to that of filters) or whether it can consider multiple notifications (thus involving both filters and patterns); and (2) whether a subscription is limited to considering a single, designated field in a notification or whether it can consider multiple fields. Expressive power is concerned with the sophistication of operators that can be used in forming subscription predicates, ranging from a simple equality predicate to expressions involving only predefined operators to expressions involving user-defined operators. As we point out in Section 5, user-defined operators suffer from the disadvantage of having arbitrary, unknown, and potentially unbounded complexity. In fact, we have observed that subscription languages with user-defined predicates are rare; in Table 3 we have combined the language classes corresponding to predefined and user-defined predicates because only a single entry, object-oriented active databases, makes use of user-defined predicates. Notice that a property of the classification in Table 4 is that the classes are inclusive: channel-based languages are a special case of subject-based languages, which in turn are a special case of content-based languages, and so on. Therefore, a technology appearing in a given row of Table 3 implicitly offers the subscription language of that row and any rows above it in the table.

We have implemented a prototype of $S_{\text {IENA }}{ }^{4}$ and used it as the event service of an agent-based, wide-area software deployment system called the Software Dock [8]. The current implementation of $S$ IENA offers two APIs, one for C++ and the other for Java. Both interfaces support the data model and subscription language described in Section 2. Two event servers are also provided by the current implementation. One (written in Java) is based on the hierarchical client/server algorithm, while the other one (written in $\mathrm{C}++$ ) is based on the acyclic peer-to-peer architecture with the subscription forwarding algorithm. These two types of servers have been used together (thus forming a hybrid topology) in the Software Dock.

Our future work will continue exploring the interplay of expressiveness and scalability, as well as other important issues concerning Internet-scale event notification such as wireless networking, application frameworks, and security. Security is a particularly intriguing issue. While traditional security techniques based on encryption can be employed in an event notification service to achieve certain kinds of security, such as authentication of publishers and integrity of notifications, the very nature of the service requires a rethinking of basic principles of secure communication. For instance, traditional notions of privacy do not apply because senders (i.e., publishers) do not designate the intended recipients (i.e, subscribers) of their messages (i.e., event notifications). Nevertheless, privacy considerations may apply in new ways, such as to prevent one client from having access to another client's subscriptions.

\section{Acknowledgments}

We would like to thank Gianpaolo Cugola, Elisabetta Di Nitto, Alfonso Fuggetta, Richard Hall, Dennis Heimbigner, and André van der Hoek for their considerable contributions in discussing and shaping many of the ideas presented in this paper.

Effort sponsored by the Defense Advanced Research Projects Agency, and Air Force Research Laboratory, Air Force Materiel Command, USAF, under agreement numbers F30602-94-C-0253, F30602-97-2-0021, F30602-98-20163 and F30602-99-C-0174; by the Air Force Office of Scientific Research, Air Force Materiel Command, USAF, under grant number F49620-98-1-0061; and by the National Science Foundation under Grant Number CCR-9701973. The U.S. Government is authorized to reproduce and distribute reprints for Governmental purposes notwithstanding any copyright annotation thereon. The views and conclusions contained herein are those of the authors and should not be interpreted as necessarily representing the official policies or endorsements, either expressed or implied, of the Defense Advanced Research Projects Agency, Air Force Researach

${ }^{4}$ http://ww.cs.colorado.edu/serl/dot/siena.html. 


\begin{tabular}{|c|c|c|c|c|}
\hline & \multicolumn{3}{|c|}{ Architecture } \\
\hline & & centralized & hierarchical client/server & general peer-to-peer \\
\hline \multirow{4}{*}{$\begin{array}{l} \\
0 \\
0\end{array}$} & channel-based & $\begin{array}{l}\text { Field [15] } \\
\text { CORBA Event Service [13] } \\
\text { Java Dist. Event Spec. [19] }\end{array}$ & CORBA Event Service [13] & $\begin{array}{l}\text { IP multicast [6] } \\
\text { iBus [18] }\end{array}$ \\
\hline & subject-based & ToolTalk [9] & $\begin{array}{c}\text { NNTP [10] } \\
\text { JEDI [5] } \\
\text { TIB/Rendezvous [20] }\end{array}$ & (none known) \\
\hline & content-based & Elvin [17] & $\begin{array}{c}\text { Keryx }[21] \\
\text { Yu et al. }[22]\end{array}$ & Gryphon [1] \\
\hline & $\begin{array}{l}\text { content-based } \\
\text { with patterns }\end{array}$ & $\begin{array}{c}\text { GEM [12] } \\
\text { Yeast [11] } \\
\text { CORBA Notification Service [14] } \\
\text { object-oriented active databases [4] }\end{array}$ & $S$ IENA & $S$ IENA \\
\hline
\end{tabular}

Table 3: A Classification of Related Technologies.

\begin{tabular}{|c|c|c|c|c|}
\hline & \multicolumn{3}{|c|}{ Scope } \\
\hline & & $\begin{array}{l}\text { Single Notification } \\
\text { Single Field }\end{array}$ & $\begin{array}{l}\text { Single Notification } \\
\text { Multiple Fields }\end{array}$ & $\begin{array}{l}\text { Multiple Notifications } \\
\text { Multiple Fields }\end{array}$ \\
\hline \multirow{3}{*}{$\begin{array}{l}0 \\
0 \\
0 \\
0\end{array}$} & $\begin{array}{r}\text { Simple } \\
\text { Equality }\end{array}$ & channel-based & (5) & 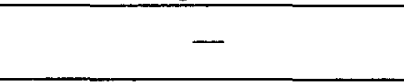 \\
\hline & $\begin{array}{r}\text { Expressions with } \\
\text { Predefined Operators }\end{array}$ & $\begin{array}{c}\text { restricted } \\
\text { subject-based }\end{array}$ & $\begin{array}{c}\text { restricted } \\
\text { content-based }\end{array}$ & $\begin{array}{c}\text { restricted } \\
\text { content-based with patterns }\end{array}$ \\
\hline & $\begin{array}{r}\text { Expressions with } \\
\text { User-defined Operators } \\
\end{array}$ & $\begin{array}{c}\text { general } \\
\text { subject-based }\end{array}$ & $\begin{array}{c}\text { general } \\
\text { content-based }\end{array}$ & $\begin{array}{c}\text { general } \\
\text { content-based with patterns }\end{array}$ \\
\hline
\end{tabular}

Table 4: Typical Features of Subscription Languages.

Laboratory or the U.S. Government.

\section{REFERENCES}

[1] G. Banavar, T. D. Chandra, B. Mukherjee, J. Nagarajarao, R. E. Strom, and D. C. Sturman. An efficient multicast protocol for content-based publish-subscribe systems. In The $19^{\text {th }}$ IEEE International Conference on Distributed Computing Systems (ICDCS '99), Austin, TX USA, May 1999.

[2] A. Carzaniga. Architectures for an Event Notification Service Scalable to Wide-area Networks. PhD thesis, Politecnico di Milano, Milano, Italy, Dec. 1998.

[3] A. Carzaniga, D. S. Rosenblum, and A. L. Wolf. Interfaces and algorithms for a wide-area event notification service. Technical Report CU-CS-888-99, Department of Computer Science, University of Colorado, Oct. 1999.

[4] S. Ceri and J. Widom. Active Database Systems: Triggers and Rules for Advanced Database Processing. Morgan Kaufmann, San Mateo, 1996.

[5] G. Cugola, E. Di Nitto, and A. Fuggetta. The JEDI event-based infrastructure and its application to the development of the OPSS WFMS. IEEE Transactions on Software Engineering, To appear.

[6] S. E. Deering and D. R. Cheriton. Multicast routing in datagram networks and extended LANs. $A C M$ Transactions on Computer Systems, 8(2):85-111, May 1990 .
[7] D. Garlan and D. Notkin. Formalizing design spaces: Implicit invocation mechanisms. In Proceedings of VDM '91: 4th International Symposium of VDM Europe on Formal Software Development Methods, pages 31-44, Noordwijkerhout, The Netherlands, Oct. 1991. Springer-Verlag.

[8] R. S. Hall, D. Heimbigner, A. van der Hoek, and A. L. Wolf. An architecture for post-development configuration management in a wide-area network. In Proceedings of the $17^{t h}$ International Conference on Distributed Computing Systems, Baltimore MD, U.S.A., May 1997.

[9] A. M. Julienne and B. Holtz. ToolTalk and open protocols, inter-application communication. Prentice-Hall, Englewood Cliffs, New Jersey, 1994.

[10] B. Kantor and P. Lapsley. Network news transfer protocol-a proposed standard for the stream-based transmission of news. Internet Requests For Comments (RFC) 977, Feb. 1986.

[11] B. Krishnamurthy and D. S. Rosenblum. Yeast: A general purpose event-action system. IEEE Transactions on Software Engineering, 21(10):845-857, Oct. 1995.

[12] M. Mansouri-Samani and M. Sloman. GEM: A generalized event monitoring language for distributed systems. IEE/IOP/BCS Distributed Systems Engineering Journal, 4(2):96-108, June 1997. 
[13] Object Management Group. CORBAservices:

Common object service specification. Technical report, Object Management Group, July 1998.

[14] Object Management Group. Notification service. Technical report, Object Management Group, Nov. 1998.

[15] S. Reiss. Connecting Tools Using Message Passing in the Field Environment. IEEE Software, pages 57-66, July 1990.

[16] D. S. Rosenblum and A. L. Wolf. A design framework for Internet-scale event observation and notification. In Proceedings of the Sixth European Software Engineering Conference, number 1301 in Lecture Notes in Computer Science, pages 344-360. Springer-Verlag, 1997.

[17] B. Segall and D. Arnold. Elvin has left the building: A publish/subscribe notification service with quenching. In Proceedings of AUUG97, Brisbane, Queensland, Australia, Sept. 3-5 1997.

[18] SoftWired AG, Zurich, Switzerland. iBus Programmer's Manual, Nov. 1998. http://www.softwired.ch/ibus.htm.

[19] Sun Microsystems, Inc., Mountain View CA, U.S.A. Java Distributed Event Specification, 1998.

[20] TIBCO Inc. Rendezvous information bus. http://www.rv.tibco.com/rvwhitepaper.html, 1996.

[21] M. Wray and R. Hawkes. Distributed virtual environments and VRML: an event-based architecture. In Proceedings of the Seventh International $W W W$ Conference (WWW7), Brisbane, Australia, 1998.

[22] H. Yu, D. Estrin, and R. Govindan. A hierarchical proxy architecture for Internet-scale event services. In Proceedings of WETICE '99, Stanford, CA, June 1999. 